\title{
Verzeichnis der Bearbeiter der 12. Auflage
}

Dr. Dietlinde Albrecht, Referentin im Innenministerium des Landes Mecklenburg-Vorpommern, Schwerin

Gerhard Altvater, Bundesanwalt beim Bundesgerichtshof, Karlsruhe

Dr. Georg Bauer, Oberstaatsanwalt beim Bundesgerichtshof, Karlsruhe

Dr. Gerhard Dannecker, Universitätsprofessor an der Ruprecht-Karls-Universität Heidelberg

Dr. Karlhans Dippel, Vorsitzender Richter am Oberlandesgericht a.D., Kronberg i.Ts.

Dr. Robert Esser, Universitätsprofessor an der Universität Passau

Dr. Klaus Geppert, em. Universitätsprofessor an der Freien Universität Berlin

Dr. Ferdinand Gillmeister, Rechtsanwalt, Freiburg

Duscha Gmel, Oberstaatsanwältin beim Bundesgerichtshof, Karlsruhe

Michael Grotz, Bundesanwalt beim Bundesgerichtshof a.D., Nationales Mitglied von Eurojust, Den Haag

Dr. Georg-Friedrich Güntge, Oberstaatsanwalt bei der Generalstaatsanwaltschaft in Schleswig Joachim Häger (, Richter am Bundesgerichtshof, Karlsruhe

Dr. Ernst-Walter Hanack, em. Universitätsprofessor an der Johannes-Gutenberg-Universität Mainz

Dr. Dr. Eric Hilgendorf, Universitätsprofessor an der Julius-Maximilians-Universität Würzburg

Dr. Dr. h.c. Thomas Hillenkamp, Universitätsprofessor an der Ruprecht-Karls-Universität Heidelberg

Dr. Tatjana Hörnle, Universitätsprofessorin an der Humboldt-Universität zu Berlin

Dr. Kristian Hohn, Wissenschaftlicher Assistent an der Bucerius Law School Hamburg

Dr. Jutta Hubrach, Richterin am Oberlandesgericht Düsseldorf

Dr. Florian Jeßberger, Universitätsprofessor an der Humboldt-Universität zu Berlin

Stefan Kirsch, Rechtsanwalt, Frankfurt am Main

Dr. Peter König, Richter am Bundesgerichtshof, Karlsruhe, Honorarprofessor an der LudwigMaximilians-Universität München

Juliane Krause, Staatsanwältin als Gruppenleiterin bei der Staatsanwaltschaft Hof

Dr. Matthias Krauß, Bundesanwalt beim Bundesgerichtshof, Karlsruhe

Dr. Christoph Krehl, Richter am Bundesgerichtshof, Karlsruhe, Honorarprofessor an der Goethe-Universität Frankfurt am Main

Perdita Kröger, Regierungsdirektorin im Bundesministerium der Justiz, Berlin

Dr. Hans Kudlich, Universitätsprofessor an der Friedrich-Alexander-Universität ErlangenNürnberg

Annette Kuschel, Richterin am Landgericht Hamburg

Heinrich Wilhelm Laufhütte, Vorsitzender Richter am Bundesgerichtshof a.D., Berlin

Dr. Hans Lilie, Universitätsprofessor an der Martin Luther-Universität Halle-Wittenberg

Dr. Manfred Möhrenschlager, Ministerialrat a.D., Bonn

Dr. Jens Peglau, Richter am Oberlandesgericht, Hamm

Dr. Andreas Popp, Universitätsprofessor an der Universität Konstanz

Dr. Ruth Rissing-van Saan, Vorsitzende Richterin am Bundesgerichtshof a.D., Bochum, Honorarprofessorin an der Ruhr-Universität Bochum

Dr. Thomas Rönnau, Universitätsprofessor an der Bucerius Law School Hamburg

Ellen Roggenbuck, Richterin am Bundesgerichtshof, Karlsruhe

Dr. Henning Rosenau, Universitätsprofessor an der Universität Augsburg

Dr. Wolfgang Ruß, Vorsitzender Richter am Bundesgerichtshof a.D., Karlsruhe 
Wilhelm Schluckebier, Richter am Bundesverfassungsgericht, Karlsruhe Johann Schmid, Oberstaatsanwalt beim Bundesgerichtshof, Karlsruhe Dr. Wilhelm Schmidt, Bundesanwalt beim Bundesgerichtshof, Karlsruhe Dr. Hendrik Schneider, Universitätsprofessor an der Universität Leipzig Dr. Heinz Schöch, Universitätsprofessor an der Ludwig-Maximilians-Universität München Dr. Dres. h.c. Friedrich-Christian Schroeder, em. Universitätsprofessor an der Universität Regensburg

Dr. Dr. h.c. mult. Bernd Schünemann, Universitätsprofessor an der Ludwig-Maximilians-Universität München

Dr. Christoph Sowada, Universitätsprofessor an der Ernst-Moritz-Arndt-Universität Greifswald Werner Theune, Richter am Bundesgerichtshof a.D., Karlsruhe

Dr. Dr. h.c. mult. Klaus Tiedemann, em. Universitätsprofessor an der Albert-Ludwigs-Universität Freiburg

Dr. Brian Valerius, Universitätsprofessor an der Universität Bayreuth

Dr. Joachim Vogel, Universitätsprofessor an der Ludwig-Maximilians-Universität München, Richter am Oberlandesgericht München

Dr. Dr. Thomas Vormbaum, Universitätsprofessor an der Fern-Universität Hagen

Dr. Tonio Walter, Universitätsprofessor an der Universität Regensburg

Dr. Thomas Weigend, Universitätsprofessor an der Universität zu Köln

Dr. Gerhard Werle, Universitätsprofessor an der Humboldt-Universität zu Berlin

Hagen Wolff, Vorsitzender Richter am Oberlandesgericht a.D., Celle

Dr. Frank Zieschang, Universitätsprofessor an der Julius-Maximilians-Universität Würzburg 